\title{
Quantitative Adjustment of the Influence of Depression on the Disabilities of the Arm, Shoulder, and Hand (DASH) Questionnaire
}

\author{
Santiago A. Lozano Calderón • David Zurakowski • \\ James S. Davis • David Ring
}

Received: 27 February 2009 / Accepted: 19 May 2009/Published online: 3 June 2009

(C) American Association for Hand Surgery 2009

\begin{abstract}
Upper extremity specific disability as measured with the Disabilities of the Arm, Shoulder and Hand (DASH) questionnaire varies more than expected based upon variations in objective impairment influenced by depression. We tested the hypothesis that adjusting for depression can reduce the mean and variance of DASH scores. Five hundred and sixteen patients (352 men, 164 women) with an average of 58 years of age (range, 18-100) were asked to simultaneously complete the DASH and Center for Epidemiologic Studies Depression Scale (CESD) scores at their initial visit to a hand surgeon. Pearson's correlations between each of the DASH items and the CESD score were obtained. The DASH score was then adjusted for the influence of Depression for women and men using ordinary least-squares regression and subtracting the product of the regression coefficient and the CES-D score from
\end{abstract}

\section{S. A. Lozano Calderón}

Department of Orthopaedic Surgery, New York Medical College,

Westchester Medical Center,

Valhalla, NY, USA

D. Zurakowski

Department of Orthopaedicsurgery, Boston Children's Hospital, Boston, MA, USA

\section{J. S. Davis}

Department of Orthopaedic Surgery, Harvard Medical School,

Massachusetts General Hospital,

Boston, MA, USA

D. Ring $(\bowtie)$

Hand and Upper Extremity Service,

Department of Orthopaedic Surgery,

Massachusetts General Hospital,

Yawkey Center Suite 2100, 55 Fruit Street,

Boston, MA 02114, USA

e-mail: dring@partners.org the raw DASH score. The average DASH score was 24 points (SD, 19; range, 0-91), and the average CES-D score was 10 points (SD, 8; range, $0-42$ ). Fourteen of the 30 items of the DASH demonstrated correlation greater than $r=0.20$. Adjustment of these DASH items for the depression effect led to significant reductions in the mean $(5.5$ points; $p<0.01)$ and standard deviation $(0.8$ points; $p<0.01)$ of DASH scores. Adjustment for depression alone had a significant but perhaps clinically marginal effect on the variance of DASH scores. Additional research is merited to determine if DASH score adjustments for the most important subjective and psychosocial aspects of illness behavior can improve correlation between DASH scores and objective impairment.

Keywords Disability · Depression · Outcomes

\section{Introduction}

The Disabilities of the Arm, Shoulder and Hand (DASH) questionnaire is the most popular measure of arm-specific health status [3, 9, 13, 17, 18, 20-23, 26, 31, 32]. The wide variation in DASH scores observed for discrete disease processes in the arm cannot be accounted for by variations in pathology or impairment alone [35-37]. Recent data have demonstrated strong correlation between depression and DASH scores [37]. If depression determines a substantial portion of arm-specific disability, then it should be possible to adjust the DASH score to account for depression and thereby decrease the mean value and/or narrow the variance of the scores. Decreased variance in measures of disability might facilitate their interpretation. In particular, decreased variance might improve correlation between impairment and disability. 
This study tested the hypothesis that adjustment of DASH scores for depression will reduce variation in the scores.

\section{Materials and Methods}

During a period of 10 months, all new adult patients in the practice of a single surgeon were asked to complete the DASH and Center for Epidemiologic Studies Depression Scale (CES-D) questionnaires as part of their initial paperwork. Completion of the forms implied consent for participation in our survey-based study. Only age and gender were recorded. No other demographic or illnessspecific data were collected. Eighty-four incomplete questionnaires were not used for a participation rate of $86 \%$. This investigation was performed with Institutional Review Board approval.

Disability of the Arm, Shoulder and Hand Questionnaire

The DASH questionnaire is a 30-item self-report questionnaire that is used to measure arm-specific disability. It was developed by the American Academy of Orthopaedic Surgeons, the Council of the Musculoskeletal Specialty Societies, and the Institute for Work and Health. Patients rate 30 tasks and symptoms using a five-point ordinal scale. The raw score is converted to a scaled score from zero to 100, with higher scores reflecting greater disability [18].

\section{Center for the Epidemiological Study of Depression}

The CES-D instrument was designed to measure a patient's current level of depressive affect. The 20 items on the questionnaire are used to measure various aspects of depression, including feelings of worthlessness, helplessness, depressed mood, lethargy, and other symptoms [34]. Each item is based on a four-point scale from zero to three, and the scale measures how often a patient felt the depressive symptom over the course of a week, with zero being "rarely or not at all" and three being "most of the time" $[1,2,8,14,34]$.

Comparisons between men and women were done to determine if the mean initial DASH, the CES-D, or the age differed between the two groups (Student's $t$ test; Table 1). Both groups were comparable in terms of depression as measured by CES-D scores but differed significantly in terms of disability (DASH score) and age (Table 1). Because of the unexpected difference in age and DASH scores between men and women, additional analyses were done to determine the correlation or influence of age over the CES-D and DASH scores (Table 1). These scores proved to be independent of age, and therefore, differences
Table 1 Age and scores for females and males.

\begin{tabular}{llll}
\hline Variable & Women & Men & Significance \\
\hline Number of patients & 164 & 352 & N/A \\
Age & & & \\
Mean & 55.4 & 60.5 & $<0.05$ \\
SD & 16.3 & 14.6 & \\
Range & $18-89$ & $23-100$ & \\
CES-D & & & \\
Mean & 9.1 & 10.2 & 0.15 \\
SD & 8.4 & 8.2 & \\
Range & $0-49$ & $0-45$ & \\
DASH & & & \\
Mean & 17.5 & 26.9 & $<0.01$ \\
SD & 16.5 & 19.2 & \\
Range & $0-89$ & $0-92$ & \\
Age vs. CES-D & $r=-0.16$, & $r=0.01$, & NS \\
correlation & $p=0.13$ & $p=0.92$ & \\
Age vs. DASH & $r=-0.09$, & $r=0.12$, & NS \\
correlation & $p=0.47$ & $p=0.15$ & \\
\hline
\end{tabular}

in terms of the initial DASH score were assumed to depend on the gender. Since gender appeared to be a modifying factor for the DASH score, statistical analyses were run independently for women and men.

\section{Statistical Analysis}

Age, DASH, and CES-D all followed a normal distribution for men and women as assessed by the KolmogorovSmirnov goodness of fit test. We measured the Pearson product-moment correlation coefficient of each item on the DASH score with the total CES-D score within each group. Items with correlations greater than $r=0.20$ (14 of 30 questions) were included in ordinary least-squares regression analyses to derive the adjustment formulas for women and men using the inverse of the regression (beta) [7] (Table 2). With this method, the product of the negative of the regression coefficient $(a)$ and the CES-D score $(b)$ was subtracted from the initial DASH score (c). The result of this subtraction: $x=c-(a \times b)$ was truncated at 0 if the result of the adjustment would have produced a negative number. The rationale of this mathematical model, based on the regression coefficients, was that a patient is predicted to a have a DASH score that is too high by that much per unit of the CES-D score; therefore, the formula adjusts down by that much the initial DASH score (Tables 3 and 4; Figs. 1 and 2).

Comparison between the initial and adjusted DASH was done for each gender to determine if the adjustment decreased in variability after removing the influence of depression (independent groups Student's $t$ test). Finally, to verify the effect of our adjustment, we ran Pearson correlations to determine the association between the 
Table 2 Pearson productmoment correlation coefficient of each item on the DASH and the CES-D total score.
Question

Pearson $r$

correlation

Significance

$(p)$
1. Open a tight or new jar.

2. Write.

3. Turn a key.

4. Prepare a meal.

5. Push open a heavy door.

6. Place an object on a shelf above your head.

7. Do heavy household chores (wash walls, wash floors).

8. Garden or do yard work.

9. Make a bed.

10. Carry a shopping bag or briefcase.

11. Carry a heavy object (over 10 pounds).

12. Change a light bulb over head.

13. Wash or blow-dry your hair.

14. Wash your back.

15. Put on a pullover sweater.

16. Use a knife to cut food.

17. Recreational activities which require little effort (card playing, knitting, etc.).

18. Recreational activities in which you take some force or impact through your arm, shoulder or hand (golf, hammering, tennis, etc.).

19. Recreational activities in which you move your arm freely (playing frisbee, badminton, etc.).

20. Manage transportation needs (getting from one place to another).

21. Sexual activities.

22. During the past week, to what extent has your arm, shoulder or hand problem interfered with your normal social activities with family, friends, neighbors or groups? (circle number)

23. During the past week were you limited in your work or other regular daily activities as a result of your arm, shoulder or hand problem? (circle number)

24. Arm, shoulder or hand pain.

25 . Arm, shoulder or hand pain when you performed any specific activity.

26. Tingling (pins and needles) in your arm, shoulder or hand.

27. Weakness in your arm, shoulder or hand.

28. Stiffness in your arm, shoulder or hand.

29. During the past week, how much difficulty have you had sleeping because of the pain in your arm, shoulder or hand? (circle number)

30. I feel less capable, less confident or less useful because of my arm, shoulder or hand problem. (circle number)

\begin{tabular}{|c|c|}
\hline 0.17 & $<0.01$ \\
\hline 0.13 & $<0.01$ \\
\hline 0.17 & $<0.01$ \\
\hline 0.13 & $<0.01$ \\
\hline 0.17 & $<0.01$ \\
\hline 0.22 & $<0.01$ \\
\hline 0.21 & $<0.01$ \\
\hline 0.21 & $<0.01$ \\
\hline 0.17 & $<0.01$ \\
\hline 0.25 & $<0.01$ \\
\hline 0.25 & $<0.01$ \\
\hline 0.23 & $<0.01$ \\
\hline 0.16 & $<0.01$ \\
\hline 0.18 & $<0.01$ \\
\hline 0.08 & 0.06 \\
\hline 0.04 & 0.36 \\
\hline 0.13 & $<0.01$ \\
\hline 0.18 & $<0.01$ \\
\hline 0.16 & $<0.01$ \\
\hline 0.22 & $<0.01$ \\
\hline 0.21 & $<0.01$ \\
\hline 0.17 & $<0.01$ \\
\hline 0.21 & $<0.01$ \\
\hline 0.23 & $<0.01$ \\
\hline 0.20 & $<0.01$ \\
\hline 0.21 & $<0.01$ \\
\hline 0.19 & $<0.01$ \\
\hline 0.23 & $<0.01$ \\
\hline 0.20 & $<0.01$ \\
\hline 0.18 & $<0.01$ \\
\hline
\end{tabular}

CES-D scores and both the initial and the adjusted DASH, expecting to find a higher correlation between the CES-D scores and the former than the CES-D and the latter (since depression was factored out). Categorical rating of the Pearson's correlation was determined by the traditional benchmarks: mild correlation, 0.10 to 0.30 ; moderate correlation, 0.31 to 0.50 ; and strong correlation, 0.51 to 1.00. Statistical analyses were performed with SPSS 14.0 statistics software (Chicago, IL, USA).

Power analysis indicated that a sample size of 150 men and 150 women would provide $90 \%$ power to detect an effect size of 0.40 between the mean and variance of the
DASH score before and after adjustment for depression (two-tailed $\alpha=0.05, \beta=0.10$; version 7.0, nQuery Advisor, Statistical Solutions, Saugus, MA, USA).

\section{Results}

Five hundred and sixteen out of 600 patients (352 men and 164 women) with an average age of 58 years (range, 18 to 100 years) completed the questionnaires (86\%). The average CES-D was ten points (SD, 8 points; range, 0 to 42 points; 95\% CI, 9-11) and the average DASH score was 
Table 3 Adjusted DASH scores for women-depression factored out.

\begin{tabular}{lrrrrrrrrr}
\hline DASH/CES-D & 0 & 5 & 10 & 15 & 20 & 25 & 30 & 35 & 40 \\
\hline 0 & 0 & 0 & 0 & 0 & 0 & 0 & 0 & 0 & 0 \\
5 & 5 & 0 & 0 & 0 & 0 & 0 & 0 & 0 & 0 \\
10 & 10 & 5 & 1 & 0 & 0 & 0 & 0 & 0 & 0 \\
15 & 15 & 10 & 6 & 1 & 0 & 0 & 0 & 0 & 0 \\
20 & 20 & 15 & 11 & 6 & 2 & 0 & 0 & 0 & 0 \\
25 & 25 & 20 & 16 & 11 & 7 & 2 & 0 & 0 & 0 \\
30 & 30 & 25 & 21 & 16 & 12 & 7 & 3 & 0 & 0 \\
35 & 35 & 30 & 26 & 21 & 17 & 12 & 8 & 3 & 0 \\
40 & 40 & 35 & 31 & 26 & 22 & 17 & 13 & 8 & 4 \\
45 & 45 & 40 & 36 & 31 & 27 & 22 & 18 & 13 & 9 \\
50 & 50 & 45 & 41 & 36 & 32 & 27 & 23 & 18 & 14 \\
55 & 55 & 50 & 46 & 41 & 37 & 32 & 28 & 23 & 19 \\
60 & 60 & 55 & 51 & 46 & 42 & 37 & 33 & 28 & 24 \\
65 & 65 & 60 & 56 & 51 & 47 & 42 & 38 & 33 & 29 \\
70 & 70 & 65 & 61 & 56 & 52 & 47 & 43 & 38 & 34 \\
75 & 75 & 70 & 66 & 61 & 57 & 52 & 48 & 43 & 39 \\
80 & 80 & 75 & 71 & 66 & 62 & 57 & 53 & 48 & 44 \\
85 & 85 & 80 & 76 & 71 & 67 & 62 & 58 & 53 & 49 \\
90 & 90 & 85 & 81 & 76 & 72 & 67 & 63 & 58 & 54 \\
95 & 95 & 90 & 86 & 81 & 77 & 72 & 68 & 63 & 59 \\
100 & 100 & 95 & 91 & 86 & 82 & 77 & 73 & 68 & 64 \\
\hline & & & & & & & & &
\end{tabular}

Table 4 Adjusted DASH scores for men-depression factored out.

\begin{tabular}{lrrrrrrrrr}
\hline DASH/CES-D & 0 & 5 & 10 & 15 & 20 & 25 & 30 & 35 & 40 \\
\hline 0 & 0 & 0 & 0 & 0 & 0 & 0 & 0 & 0 & 0 \\
5 & 5 & 3 & 0 & 0 & 0 & 0 & 0 & 0 & 0 \\
10 & 10 & 8 & 5 & 3 & 1 & 0 & 0 & 0 & 0 \\
15 & 15 & 13 & 10 & 8 & 6 & 3 & 1 & 0 & 0 \\
20 & 20 & 18 & 15 & 13 & 11 & 8 & 6 & 4 & 1 \\
25 & 25 & 23 & 20 & 18 & 16 & 13 & 11 & 9 & 6 \\
30 & 30 & 28 & 25 & 23 & 21 & 18 & 16 & 14 & 11 \\
35 & 35 & 33 & 30 & 28 & 26 & 23 & 21 & 19 & 16 \\
40 & 40 & 38 & 35 & 33 & 31 & 28 & 26 & 24 & 21 \\
45 & 45 & 43 & 40 & 38 & 36 & 33 & 31 & 29 & 26 \\
50 & 50 & 48 & 45 & 43 & 41 & 38 & 36 & 34 & 31 \\
55 & 55 & 53 & 50 & 48 & 46 & 43 & 41 & 39 & 36 \\
60 & 60 & 58 & 55 & 53 & 51 & 48 & 46 & 44 & 41 \\
65 & 65 & 63 & 60 & 58 & 56 & 53 & 51 & 49 & 46 \\
70 & 70 & 68 & 65 & 63 & 61 & 58 & 56 & 54 & 51 \\
75 & 75 & 73 & 70 & 68 & 66 & 63 & 61 & 59 & 56 \\
80 & 80 & 78 & 75 & 73 & 71 & 68 & 66 & 64 & 61 \\
85 & 85 & 83 & 80 & 78 & 76 & 73 & 71 & 69 & 66 \\
90 & 90 & 88 & 85 & 83 & 81 & 78 & 76 & 74 & 71 \\
95 & 95 & 93 & 90 & 88 & 86 & 83 & 81 & 79 & 76 \\
100 & 100 & 98 & 95 & 93 & 91 & 88 & 86 & 84 & 81 \\
\hline
\end{tabular}

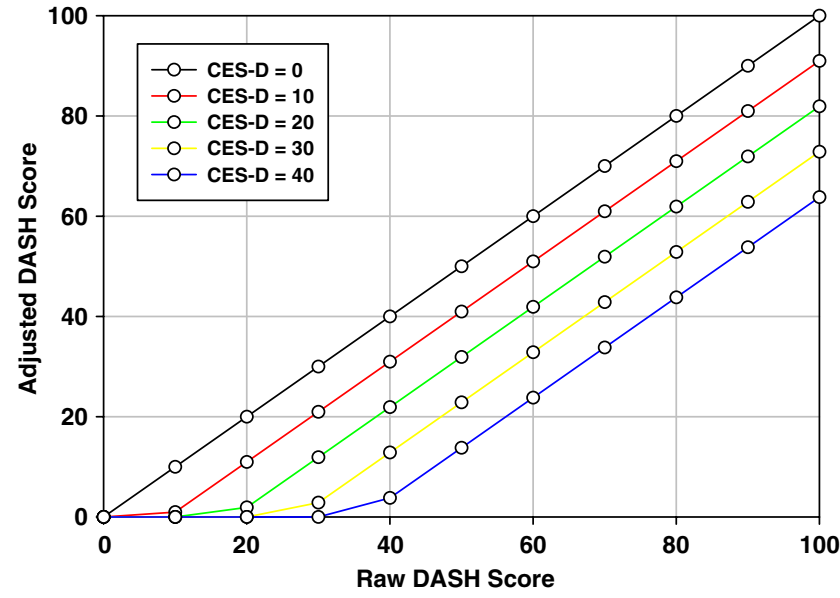

Figure 1 Comparison between the initial and the adjusted DASH in females.

24 points (SD, 19 points; range, 0 to 91; 95\% CI, 22-26). The mean depression-adjusted DASH score was 18.5 points (SD, 18 points; range, 0 to 88 ; 95\% CI, 17-20).

The decrease of 5.5 points in the average depressionadjusted DASH score as compared to the initial DASH score was expected based upon the lowered weighting of the answers on 14 questions and was statistically significant $(t=17.4, p<0.01)$. The linear regression equations to predict DASH scores from CES-D scores were:

Women $: y=0.905 \times \mathrm{CES}-\mathrm{D}+9.28$.

The regression coefficient for CES-D was highly significant $(t=6.59, p<0.0001)$.

Men $: y=0.468 \times \mathrm{CES}-\mathrm{D}+22.15$.

The regression coefficient for CES-D was highly significant $(t=3.75, p<0.001)$.

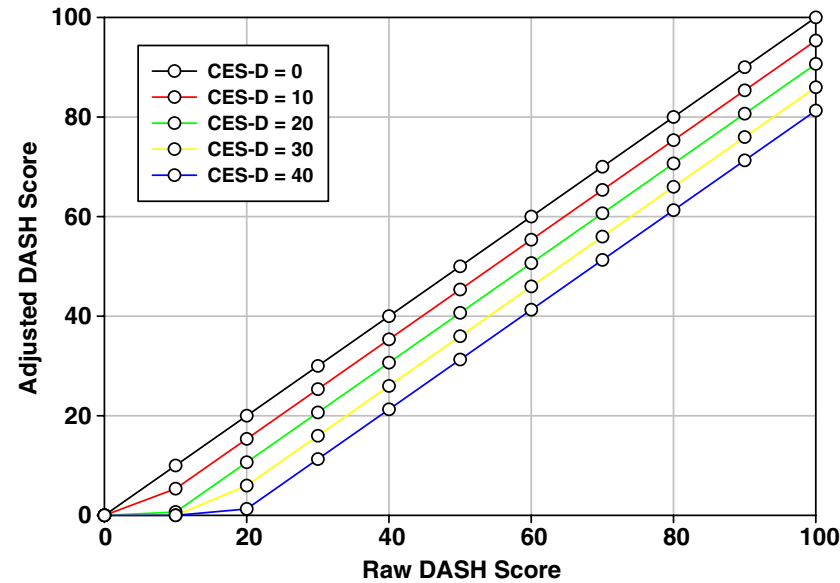

Figure 2 Comparison between the initial and the adjusted DASH in males. 
The calculated adjustment formulas were:

$$
\begin{aligned}
& \text { Adjusted DASH score }=\text { Initial DASH Score } \\
& -(0.905 \times \text { CES-D }) \text { for women and Adjusted } \\
& \text { DASH score }=\text { Initial DASH Score } \\
& -(0.468 \times \text { CES }-D) \text { for men. }
\end{aligned}
$$

\section{Analysis by Gender}

The CES-D scores were comparable between both genders ( 9 vs. 10 points, respectively, $p=0.15$ ). The initial DASH score was significantly greater for men before (27 vs. 18 points, $t=-5.1, p<0.001)$ and after adjusting for depression (22 vs. 11 points, $t=-6.6, p<0.001$ ). The reduction of the mean after the adjustment was statistically significant for both groups. Women's reduction averaged seven points $(t,-18.1 ; p<0.001)$, while men's averaged five points $(t$, $21.2 ; p<0.01)$. The standard deviation was also analyzed since it is an indirect measure of variation. The larger the standard deviation of the DASH score, the wider the confidence interval in consideration and the larger distance between the values to the mean DASH, in other words, the greater the DASH score variation. The standard deviation of the DASH scores decreased in both women and men when adjusting for depression (narrower confidence interval, subsequent decrease of variation). Despite being statistically significant, this reduction was perhaps clinically marginal: two points in women (from 17 to 15 points, $p<$ 0.01 ) and one point in men (19 to 18 points, $p<0.01$ ).

There was a significant moderate correlation between the initial DASH score and the CES-D in women $(r=0.45, p<$ $0.01)$. There was no correlation between the adjusted DASH and the CES-D $(r=-0.01, p=0.84)$, confirming successful correction with our adjusted model in this group. Correlation between the initial and the corrected DASH was strong and significant in women $(r=0.77)$.

There was a significant but more limited correlation between the initial DASH score and the CES-D in men (Pearson $r=0.25, p<0.01$ ) compared to that observed in women. There was no correlation between the adjusted DASH and CES-D ( $r=0.01, p=0.82)$, confirming successful correction also in men. Correlation between the initial and the corrected DASH was also significant in this group $(r=0.98, p<0.01)$.

\section{Discussion}

According to the World Health Organization, impairments are the manifestations of an underlying pathology and represent objective, physical deviation or loss due to problems in bodily function or structure. Disability refers more generally to activity limitations, whether these are the result of objective physical impairments or psychosocial factors [43]. For some illnesses-stroke, renal end-stage disease, chronic high-resistance flow respiratory disorders, diabetes, among others - there is a strong correlation between impairment and disability. For instance, several papers have documented a substantial correlation between motor impairment and disability after stroke [11, 16, 30, 33, $38,42]$. On the other hand, the correlation between musculoskeletal impairment and disability in the case of musculoskeletal illness in particular is consistently weak. For example, Mock et al. in 2000 analyzed the determinants of disability in 302 patients with fractures of the lower extremity and found that physical impairment was a weak predictor of disability; the most important factors according to this investigation were age, socioeconomic status, preinjury health, and social support [29]. Others have demonstrated low socioeconomic status and older age as strong factors predicting increased levels of disability for various illnesses $[6,15,25,27,28,44]$. One measure of the disparity between disability and impairment is the greater variation in measure of disability than it would be expected based upon variations seen in impairment.

We chose to focus on depression because Jones and colleagues [24] among others have identified depression as one of the strongest predictors of disability in populations with a variety of medical impairments such as renal, pulmonary, or rheumatologic diseases [4-6, 12, 35-37]. The rule holds for arm-specific disability, where recent work has emphasized the influence of depression and other psychological factors on arm-specific disability as measured by the DASH [10, 35-37].

While we did observe a significant decrease in both the mean and standard deviation of DASH scores by adjusting for the affect of depression, the decrease in variation was very small. Furthermore, the DASH and depressionadjusted DASH scores were highly correlated, suggesting that adjusting for depression did not provide a clear advantage or notable distinction from unadjusted DASH scores. Consequently, it must be concluded that disability is sufficiently complex that adjustment for one strong correlate (in this case depression) can significantly, but only marginally, decrease the variation in disability scores.

As further evidence of the psychosocial aspects of illness behavior in general and disability in particular, we found that gender had a significant influence on disability. Moreover, the effect of depression on DASH was different for men and women. Differences in the experience of pain and disability between men and women have been observed fairly consistently [19, 39-41].

Our study should be interpreted in light of the use of mixed diagnoses and illness stages, no attempt to account for disease and impairment, and the need to discard incomplete forms, since patients that complete the forms 
may be different from those that do not complete the forms in very important ways. Nonetheless, the fact that we were able to decrease the mean and variation of the DASH scores significantly by adjusting for depression in this broad sample of hand surgery patients promotes the value of considering the psychosocial aspects of illness behavior and of continuing research to understand the differences between impairment and disability. The fact that the changes in scores were marginal and of questionable relevance underscores the complexity of disability and the need for additional research.

\section{References}

1. Baldwin G, Shean GD. A taxometric study of the center for epidemiological studies depression scale. Genet Soc Gen Psychol Monogr. 2006;132(2):101-28. doi:10.3200/MONO.132.2.101128.

2. Batistoni SS, Neri AL, Cupertino AP. Validity of the center for epidemiological studies depression scale among Brazilian elderly. Rev Saude Publica. 2007;41(4):598-605. doi:10.1590/S003489102007000400014.

3. Beaton DE, Katz JN, Fossel AH, Wright JG, Tarasuk V, Bombardier C. Measuring the whole or the parts? Validity, reliability, and responsiveness of the disabilities of the arm, shoulder and hand outcome measure in different regions of the upper extremity. J Hand Ther. 2001;14(2):128-46.

4. Beckham JC, D'Amico CJ, Rice JR, Jordan JS, Divine GW, Brook WB. Depression and level of functioning in patients with rheumatoid arthritis. Can J Psychiatry. 1992;37(8):539-43.

5. Brooks WB, Jordan JS, Divine GW, Smith KS, Neelon FA. The impact of psychologic factors on measurement of functional status. Assessment of the sickness impact profile. Med Care. 1990;28(9):793-804. doi:10.1097/00005650-199009000-00009.

6. Butcher JL, MacKenzie EJ, Cushing B, Jurkovich G, Morris J, Burgess A, et al. Long-term outcomes after lower extremity trauma. J Trauma. 1996;41(1):4-9. doi:10.1097/00005373-19960 7000-00002.

7. Chatterjee SH. Regression analysis by example. 4th ed. New York: John Wiley; 2006.

8. Cheung YB, Liu KY, Yip PS. Performance of the CES-D and its short forms in screening suicidality and hopelessness in the community. Suicide Life Threat Behav. 2007;37(1):79-88. doi:10. 1521/suli.2007.37.1.79.

9. Dowrick AS, Gabbe BJ, Williamson OD, Cameron PA. Does the disabilities of the arm, shoulder and hand (DASH) scoring system only measure disability due to injuries to the upper limb? J Bone Jt Surg Br. 2006;88(4):524-7. doi:10.1302/0301-620X.88B4. 17223.

10. Droll KP, Potter J, Harniman E, Schemitssch EH, McKee MD. Outcomes following plate fixation of fractures of both bones of the forearm in adults. J Bone Joint Surg Am. 2007;89(12):2619 24.

11. Gowland C, Stratford P, Ward M, Moreland J, Torresin W, Van Hullenaar S, et al. Measuring physical impairment and disability with the Chedoke-McMaster Stroke Assessment. Stroke. 1993;24 (1):58-63

12. Green MM, McFarlane AC, Hunter CE, Griggs WM. Undiagnosed post-traumatic stress disorder following motor vehicle accidents. Med J Aust. 1993;159(8):529-34.
13. Gummesson C, Atroshi I, Ekdahl C. The Disabilities of the Arm, Shoulder and Hand (DASH) outcome questionnaire: longitudinal construct validity and measuring self-rated health change after surgery. BMC Musculoskelet Disord. 2003;4:11. doi:10.1186/ 1471-2474-4-11.

14. Hann D, Winter K, Jacobsen P. Measurement of depressive symptoms in cancer patients: evaluation of the Center for Epidemiological Studies Depression Scale (CES-D). J Psychosom Res. 1999;46(5):437-43. doi:10.1016/S0022-3999(99)00004-5.

15. Harris LE, Luft FC, Rudy DW, Tierney WM. Clinical correlates of functional status in patients with chronic renal insufficiency. Am J Kidney Dis. 1993;21(2):161-6.

16. Harvey RL, Roth EJ, Heinemann AW, Lovell LL, McGuire JR, Diaz S. Stroke rehabilitation: clinical predictors of resource utilization. Arch Phys Med Rehabil. 1998;79(11):1349-55. doi:10. 1016/S0003-9993(98)90226-X.

17. Hervas MT, Navarro Collado MJ, Peiro S, Rodrigo Perez JL, Lopez Mateu P, Martinez Tello I. Spanish version of the DASH questionnaire. Cross-cultural adaptation, reliability, validity and responsiveness. Med Clin (Barc). 2006;127(12):441-7. doi:10. $1157 / 13093053$.

18. Hudak PL, Amadio PC, Bombardier C. Development of an upper extremity outcome measure: the DASH (disabilities of the arm, shoulder and hand) [corrected]. The Upper Extremity Collaborative Group (UECG). Am J Ind Med. 1996;29(6):602-8. doi:10.1002/(SICI)1097-0274(199606)29:6<602::AID-AJIM4> 3.0.CO;2-L.

19. Hurley RW, Adams MC. Sex, gender, and pain: an overview of a complex field. Anesth Analg. 2008;107(1):309-17. doi:10.1213/ 01.ane.0b013e31816ba437.

20. Imaeda $T$, Toh $S$, Nakao $Y$, Nishida J, Hirata $H$, Ijichi M, et al. Validation of the Japanese Society for Surgery of the Hand version of the Disability of the Arm, Shoulder, and Hand questionnaire. J Orthop Sci. 2005;10(4):353-9. doi:10.1007/s00776-005-0917-5.

21. Jester A, Harth A, Germann G. Measuring levels of upperextremity disability in employed adults using the DASH Questionnaire. J Hand Surg [Am]. 2005;30(5):1074 e1-e10.

22. Jester A, Harth A, Wind G, Germann G, Sauerbier M. Disabilities of the arm, shoulder and hand (DASH) questionnaire: Determining functional activity profiles in patients with upper extremity disorders. J Hand Surg [Br]. 2005;30(1):23-8. doi:10.1016/j. jhsb.2004.08.008.

23. Jester A, Harth A, Wind G, Germann G, Sauerbier M. Does the disability of shoulder, arm and hand questionnaire (DASH) replace grip strength and range of motion in outcomeevaluation? Handchir Mikrochir Plast Chir. 2005;37(2):126-30. doi:10.1055/s-2004-821279.

24. Jones PW, Baveystock CM, Littlejohns P. Relationships between general health measured with the sickness impact profile and respiratory symptoms, physiological measures, and mood in patients with chronic airflow limitation. Am Rev Respir Dis. 1989;140(6):1538-43.

25. Julius M, Hawthorne VM, Carpentier-Alting P, Kneisley J, Wolfe RA, Port FK. Independence in activities of daily living for endstage renal disease patients: biomedical and demographic correlates. Am J Kidney Dis. 1989;13(1):61-9.

26. Lee EW, Lau JS, Chung MM, Li AP, Lo SK. Evaluation of the Chinese version of the Disability of the Arm, Shoulder and Hand (DASH-HKPWH): cross-cultural adaptation process, internal consistency and reliability study. J Hand Ther. 2004;17(4):41723.

27. MacKenzie EJ, Shapiro S, Smith RT, Siegel JH, Moody M, Pitt A. Factors influencing return to work following hospitalization for traumatic injury. Am J Public Health. 1987;77(3):329-34. doi:10.2105/AJPH.77.3.329. 
28. Mitchell BD, Stern MP, Haffner SM, Hazuda HP, Patterson JK. Functional impairment in Mexican Americans and non-Hispanic whites with diabetes. J Clin Epidemiol. 1990;43(4):319-27. doi:10.1016/0895-4356(90)90118-9.

29. Mock C, MacKenzie E, Jurkovich G, Burgess A, Cushing B, deLateur B, et al. Determinants of disability after lower extremity fracture. J Trauma. 2000;49(6):1002-11. doi:10.1097/00005373200012000-00005.

30. Nas K, Gur A, Cevik R, Sarac AJ. The relationship between physical impairment and disability during stroke rehabilitation: effect of cognitive status. Int J Rehabil Res. 2004;27(3):181-4. doi:10.1097/00004356-200409000-00002.

31. Offenbacher M, Ewert T, Sangha O, Stucki G. Validation of a German version of the 'Disabilities of Arm, Shoulder and Hand' questionnaire (DASH-G). Z Rheumatol. 2003;62(2):168-77. doi:10.1007/s00393-003-0461-7.

32. Orfale AG, Araujo PM, Ferraz MB, Natour J. Translation into Brazilian Portuguese, cultural adaptation and evaluation of the reliability of the Disabilities of the Arm, Shoulder and Hand Questionnaire. Braz J Med Biol Res. 2005;38(2):293-302. doi:10.1590/S0100-879X2005000200018.

33. Pallicino P, Snyder W, Granger C. The NIH stroke scale and the FIM in stroke rehabilitation. Stroke. 1992;23(6):919.

34. Radloff LS. The CES-D scale: a self-report depression scale for research in the general population. Appl Psychol Meas. 1977;1 (3):385-401. doi:10.1177/014662167700100306.

35. Ring D, Guss D, Malhotra L, Jupiter JB. Idiopathic arm pain. J Bone Jt Surg Am. 2004;86-A(7):1387-91.

36. Ring D, Kadzielski J, Malhotra L, Lee SG, Jupiter JB. Psychological factors associated with idiopathic arm pain. J Bone Jt Surg Am. 2005;87(2):374-80. doi:10.2106/JBJS.D.01907.
37. Ring D, Kadzielski J, Fabian L, Zurakowski D, Malhotra LR, Jupiter JB. Self-reported upper extremity health status correlates with depression. J Bone Jt Surg Am. 2006;88(9):1983-8. doi:10.2106/JBJS.E.00932.

38. Roth EJ, Heinemann AW, Lovell LL, Harvey RL, McGuire JR, Diaz S. Impairment and disability: their relation during stroke rehabilitation. Arch Phys Med Rehabil. 1998;79(3):329-35. doi:10.1016/S0003-9993(98)90015-6.

39. Sa KN, Baptista AF, Matos MA, Lessa I. Chronic pain and gender in Salvador population, Brazil. Pain. 2009 Jul 29.

40. Singh JA, Gabriel S, Lewallen D. The impact of gender, age, and preoperative pain severity on pain after TKA. Clin Orthop Relat Res. 2008;466(11):2717-23.

41. Sonoda S, Chino N, Domen K, Saitoh E. Changes in impairment and disability from the third to the sixth month after stroke and its relationship evaluated by an artificial neural network. Am J Phys Med Rehabil. 1997;76(5):395-400. doi:10.1097/00002060199709000-00010.

42. The World Health Organization International Classification of Impairment, Disability and Handicap: A Manual of Classification Relating to the Consequence of Disease. The World Health Organization. 1980.

43. Yanagita M, Willcox BJ, Masaki KH, Chen R, He Q, Rodriguez $\mathrm{BL}$, et al. Disability and depression: investigating a complex relation using physical performance measures. Am J Geriatr Psychiatry. 2006;14(12):1060-8. doi:10.1097/01.JGP.00002243 64.70515.12.

44. Yang MS, Tseng CK, Chung HH, Yip WH. The functional assessment of chronic pain patients activity of daily living and psychosocial disability. Gaoxiong Yi Xue Ke Xue Za Zhi. 1992;8 (10):552-61. 